\section{Occurrences of ochratoxin A in slaughtered wild boar (Sus scrofa)}

\author{
Giancarlo Bozzo, Edmondo Ceci, \\ Elisabetta Bonerba, Angela Di Pinto, \\ Gaetano Vitale Celano, \\ Giuseppina Tantillo \\ Dipartimento di Sanità Pubblica e \\ Zootecnia, Università degli Studi di Bari, \\ Valenzano (BA), Italy
}

\begin{abstract}
Ochratoxins are fungal secondary metabolites that may contaminate a broad variety of foodstuffs, such as grains, vegetables, coffee, dried fruits, beer, wine and meats. Ochratoxins are considered powerful nephrotoxins, carcinogens, teratogens in rats and likely in humans. In 2011, during a programme aimed to survey the presence of ochratoxin A in 35 regularly slaughtered wild boars in Calabria region (Southern Italy), ochratoxin A (OTA) was detected in 35 kidneys, 33 urinary bladders, 33 livers and 32 muscles of 35 animals at the following levels: $1.05 \mathrm{ppb}$ (0.1-3.9 ppb), 0.5 ppb [not detected (ND)-2.6 ppb], 0.4 ppb (ND$2 \mathrm{ppb}$ ), $0.2 \mathrm{ppb}$ (ND-0.5 ppb), respectively. A total of 12 samples of kidney, 4 samples of liver, and 4 samples of urinary bladder showed levels of OTA higher than the level (1 ppb) established by the guidelines of the Italian Ministry of Health circular No. 10.
\end{abstract}

\section{Introduzione}

L'ocratossina A (OTA) è il prodotto del metabolismo secondario di alcune specie di funghi microscopici (Aspergillus spp. e Penicillium spp.) che, se presenti su cereali, semi oleaginosi, frutta secca ed essiccata, legumi, spezie, caffè, cacao, trovano il substrato ideale per la loro moltiplicazione a condizioni di temperatura ed umidità favorevoli (Delage et al., 2003; Peterson, 2000). L'OTA è una molecola caratterizzata da elevata stabilità e negli alimenti, se conservati per lunghi periodi, non subisce alcun tipo di degradazione; i sistemi chimici, fisici e biologici messi a punto per la detossificazione delle derrate alimentari non hanno trovano finora una reale applicazione pratica (Brown et al., 1998).

L'OTA può, pertanto, contaminare direttamente alimenti di origine vegetale, soprattutto i mangimi, e risultare un contaminante per gli alimenti di origine animale e per i prodotti derivati.
Considerata un potente nefrotossico, cancerogeno, teratogeno, ed immunogeno per gli animali e per l'uomo (Pfohl-Leszkowicz e Manderville 2007), l'OTA esercita la propria azione tossica attraverso diversi meccanismi: inibizione della sintesi proteica, perossidazione lipidica con modificazioni della permeabilità di membrana, alterazione nella produzione di ATP e danneggiamento degli acidi nucleici (Schaaf et al., 2002).

Gli animali da reddito mostrano differente sensibilità all'azione dell'OTA in relazione alla dose assunta, alla durata dell'esposizione, all'età, al sesso, allo stato fisiologico e all'eventuale sinergismo d'azione con altre micotossine eventualmente presenti nei mangimi. In tutti gli animali da reddito la micotossina tende prevalentemente ad accumularsi a livello dell'apparato renale ed epatico e in concentrazioni minori nel tessuto muscolare (0'Brien e Dietrich, 2005).

Il rischio sanitario che l'OTA rappresenta per l'uomo ha indotto l'Unione Europea ad introdurre, nell'ambito del Regolamento CE n. 1881/2006 (Commissione Europea, 2006), limiti massimi per l'OTA in diversi alimenti, soprattutto di origine vegetale, senza tuttavia indicare il limite ammissibile nelle carni e nei prodotti derivati; tuttavia il Ministero della Sanità, con l'emanazione della circolare n.10 del 9 giugno 1999 (Ministero della Sanità, 1999), invece, ha fissato il limite massimo di 1 ppb per l'OTA nelle carni di suino e nei derivati. I numerosi dati bibliografici oggi disponibili, che descrivono la presenza di OTA in diverse specie di animali da reddito e non (PfohlLeszkowicz e Manderville 2007; Abarca et al., 2001) tuttavia, non hanno considerato il cinghiale (sus scrofa), che in alcune regioni italiane viene allevato, non esclusivamente per il consumo delle carni fresche. La nostra indagine, frutto di una collaborazione tra la Sezione di Sicurezza degli Alimenti del Dipartimento di Sanità Pubblica e Zootecnia dell'Università degli Studi di Bari e l'ASP di Reggio Calabria, ha lo scopo di valutare il livello di contaminazione di OTA in tessuti e organi di cinghiali regolarmente macellati, evidenziarne le lesioni macroscopiche e condurre indagini istologiche, al fine di correlare la presenza della micotossina con le alterazioni rilevate.

\section{Materiali e Metodi}

L'indagine è stata condotta su 35 cinghiali allevati allo stato brado all'interno di aree boschive costituite in prevalenza da querce e condotti a regolare macellazione nell'anno 2011. Da ciascuna carcassa, dopo l'esame ispettivo post mortem sono stati prelevati rene, vescica, fegato e parte del longissimus dorsi.

I campioni raccolti sono stati immediata-
Correspondence: Giancarlo Bozzo, Dipartimento di Sanità Pubblica e Zootecnia, Università degli Studi di Bari, strada provinciale per Casamassima km 3, 70010 Valenzano (BA), Italy. Tel. +39.080.5443850 - Fax: +39.080 .5443855 .

Email: giancarlo.bozzo@uniba.it

Key words: HPLC, OTA, Sus scrofa.

Conflict of interests: the authors declare no potential conflict of interests.

Received for publication: 15 January 2013.

Revision received: 4 July 2013.

Accepted for publication: 4 July 2013.

This work is licensed under a Creative Commons Attribution 3.0 License (by-nc 3.0).

(C) Copyright G. Bozzo et al., 2013

Licensee PAGEPress, Italy

Italian Journal of Food Safety 2013; 2:e39

doi:10.4081/ijfs.2013.e39

mente refrigerati ed inviati presso i laboratori della sezione di Sicurezza degli Alimenti ed in questa sede sono stati sottoposti ad un più approfondito esame macroscopico. Dai campioni di rene, fegato e vescica sono state allestite 2 aliquote, una destinata alle indagini chimiche, l'altra destinata alle indagini istologiche; quest'ultima è stata fissata in formalina tamponata al $10 \%$. I campioni di tessuto muscolare sono stati sottoposti esclusivamente ad indagine chimica.

L'esame istologico ha previsto una prima fase di inclusione dei campioni in paraffina, quindi gli inclusi sono stati processati con microtomo rotativo eseguendo un taglio di 4 $\mu \mathrm{m}$ ed in ultimo colorati con ematossilina eosina. Per la ricerca dei residui di dell'OTA tutti i campioni, sono stati processati per essere sottoposti ad analisi quali-quantitativa con metodo cromatografico ad alta risoluzione high performance liquid cromatography (HPLC).

L'esame chimico ha previsto l'esecuzione di diverse fasi di lavorazione. Inizialmente, si è effettuata la preparazione del campione: $20 \mathrm{~g}$ di tessuto sono stati omogenati con $6 \mathrm{~mL}$ di acido fosforico $1 \mathrm{M}$ per alcuni minuti. Due grammi e mezzo dell'omogenato ottenuto sono stati centrifugati per 5 min a 4000 rpm dopo aggiunta di $5 \mathrm{~mL}$ di acetato di etile. Al surnatante, per l'estrazione in fase solida, sono stati aggiunti 6 $\mathrm{mL}$ di $\mathrm{NaHCO}_{3}$ 0,5 M (pH 8.4). Successivamente si è provveduto all'estrazione su fase solida (SPE) e purificazione del campione mediante colonnine di immunoaffinità: l'estratto purificato è stato caricato su colonnine di immunoaffinità Ochratest $^{\mathrm{TM}}$ (VICAM, Milford, MA USA) che permettono il legame selettivo tra l'OTA e specifici anticorpi monoclonali. Per il caricamento è stato rispettato un 
flusso di 2-3 gocce/sec, come richiesto nella metodica della casa produttrice (VICAM). La colonnina, caricata del campione, è stata lavata due volte con $10 \mathrm{~mL}$ di acqua deionizzata e $10 \mathrm{~mL}$ di PBS sempre alla velocità di 2-3 gocce/sec e asciugata con un flusso di aria. L'OTA presente nel campione è stata eluita dalla colonnina con 1,5 mL di metanolo puro (Carlo Erba Reagents, Milano, Italia) garantendo un tempo minimo di contatto tra colonnina e metanolo di circa 60 secondi e mantenendo la velocità di flusso di 1 goccia/sec. Infine, si è passati alla determinazione analiti$c a$ : l'analisi cromatografica è stata effettuata mediante cromatografo liquido ad alta efficienza (Agilent Technologies Ltd., Santa Clara, CA, USA) modello 1100 composto dal modulo di degassazione della fase mobile, dal modulo della pompa, dal modulo dell'auto-campionatore in cui è alloggiato l'iniettore Rheodyne con loop da 100 L e dal modulo di alloggiamento della colonna analitica per la termostatazione della stessa. La separazione cromatografica è stata eseguita su colonna analitica Restek RP-C18 (5 m, 250 mm x 4,6 mm diametro interno). Come rivelatore è stato utilizzato un detector a fluorescenza (Mod. 1100; Agilent Technologies Ltd., Santa Clara, CA, USA) con gestione del segnale ottenuto con software BIORAD. L'eluizione isocratica è stata effettuata termostatando la colonna alla temperatura di $30^{\circ} \mathrm{C}$, con flusso di $1,2 \mathrm{~mL} / \mathrm{min}$. La fase mobile è costituita da acqua:acetonitrile: acido acetico (99:99:2) mentre il detector a fluorescenza è stato settato su una lunghezza d'onda di eccitazione a $333 \mathrm{~nm}$ e di emissione a $477 \mathrm{~nm}$ (Monaci et al., 2004).

\section{Risultati}

Le analisi chimiche condotte hanno consentito di rilevare la presenza di OTA in tutti i reni esaminati; in 33 campioni di fegato (fatta eccezione dei campioni 24 e 34); in 33 campioni di vescica urinaria (fatta eccezione dei campioni 34 e 35) e in 32 campioni di tessuto muscolare (fatta eccezione dei campioni 9, 34 e 35). Il valore medio riscontrato per il tessuto renale è stato di $1,05 \mathrm{ppb}(0,1-3,9 \mathrm{ppb})$, di 0,4 ppb nel fegato [not detected (ND)-2 ppb], di $0,5 \mathrm{ppb}$ (ND-2,6 ppb) nella vescica urinaria e di $0,2 \mathrm{ppb}$ (ND-0,5 ppb) nel muscolo (Figura 1).

In 12 campioni di rene, 4 di fegato e 4 di vescica urinaria dei 35 animali sottoposti ad analisi il quantitativo di OTA è risultato più elevato rispetto a quanto stabilito dalla circolare n.10 del 9 giugno 1999 (1 ppb) (Ministero della Sanità, 1999) (Figura 1).

Le indagini anatomo-istolo-patologiche hanno consentito di rilevare lesioni nei campioni di rene che presentavano una concentrazione di 0TA>3,1 ppb (campioni 6, 16, 20, 33), nel campione di fegato con concentrazione di OTA pari a 1,8 ppb (campione 6) e nei campioni di vescica urinaria con concentrazione di OTA $>1,7$ ppb (campioni 6 e 20).

\section{Rene: risultati macroscopici e istologici}

All'esame macroscopico i reni dei campioni 6, 16, 20 e 33 pur non presentando alterazioni di volume, apparivano pallidi e con piccoli focolai di fibrosi corticale. Al taglio la porzione corticale era pallida ed il rapporto tra la porzione corticale e quella midollare era in favore della prima; a confermare il danno renale è stato possibile, inoltre, osservare lo slargamento del bacinetto renale (Figura 2).

L'esame istologico ha permesso di confermare la presenza di diffusa fibrosi e gravi alterazioni morfo-funzionali dei glomeruli e dei tubuli renali. I glomeruli apparivano interessati da un processo degenerativo atrofico, aumento dello spazio sub-capsulare e ispessimento della capsula di Bowman; intorno ad essi era apprezzabile una notevole componente infiammatoria caratterizzata da cellule principalmente di tipo fibroblastico. Il processo infiammatorio cronico coinvolgeva anche $\mathrm{i}$ tubuli renali, che risultavano poco visibili all'interno del tessuto neoformato; le cellule dei tubuli prossimali erano alterate nella

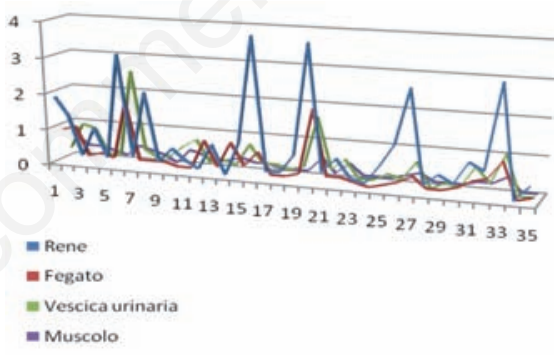

Figura 1. Grafico a linee relativo ai valori di ocratossina A nei campioni esaminati espressi in $\mathrm{ppb}$.

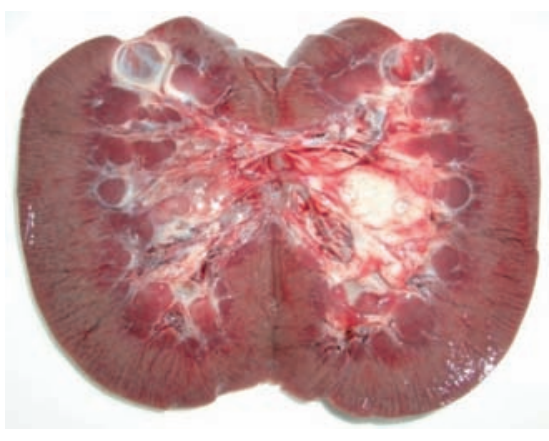

Figura 2. Rene: pallore; alterazione del rapporto fra corticale e midollare; allargamento del bacinetto renale. forma ed era presente vacuolizzazione citoplasmatica, caratteristica tipica della tubulonefrosi (Figura 3).

\section{Fegato: risultati macroscopici e istologici}

Il fegato che presentava una concentrazione di OTA pari a 1,8 ppb, pur conservando il volume normale, presentava lobuli epatici particolarmente evidenti ed i margini dell'organo si presentavano assottigliati. In superficie sono state riscontrate zone di colore rosso scuro alternate a zone decisamente più chiare $\mathrm{e}$, di queste, alcune risultavano più pallide della norma. Il taglio di alcune zone del parenchima epatico è risultato difficoltoso ed ha comportato colio di sangue mettendo in luce aree di iperemia. All'esame istologico è stato possibile apprezzare ampie zone di degenerazione ed un quadro di lieve steatosi, per la presenza di cellule ad anello con nucleo periferico e citoplasma occupato da un grosso vacuolo otticamente vuoto (Figura 4). Lo spazio tra i sinusoidi, inoltre, risultava aumentato. Intorno ai vasi sanguigni ed ai dotti biliari sono stati osservati un notevole numero di fibroblasti ed è stato possibile evidenziare aree nodulari interessate da fenomeni di fibrosi in prossimità di vasi.

I campioni di vescica urinaria con concentrazione di 0TA $>1,7 \mathrm{ppb}$ presentavano la pare-

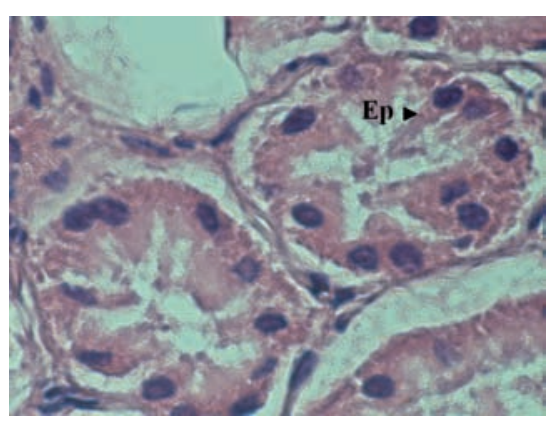

Figura 3. Rene: tubulonefrosi caratterizzata da presenza di epiteli (Ep) allungati e vacuolizzazione (colorazione ematossilina eosina, ingrandimento $100 \mathrm{x}$ ).

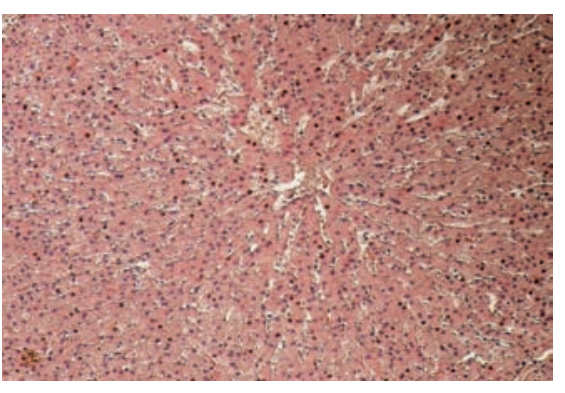

Figura 4. Fegato: aspetto istologico del lobulo epatico (colorazione ematossilina eosina, ingrandimento $20 \mathrm{x}$ ). 
te più spessa rispetto al normale (Figura 5), la mucosa era tumida e superficialmente era possibile osservare abbondante presenza di muco. Sulla superficie interna si osservava arrossamento diffuso, indice di fenomeni iperemici associati a lievi emorragie puntiformi.

Attraverso l'esame istologico è stata evidenziata proliferazione connettivale diffusa che giustificava l'ispessimento della parete, apprezzabile anche macroscopicamente. È stato altresì possibile evidenziare uno stato di sofferenza della mucosa e in particolare un quadro degenerativo dell'epitelio vescicale con estese vacuolizzazioni a livello citoplasmatico (Figura 6).

\section{Discussione}

La più alta concentrazione di OTA si osserva nel rene e in misura minore, nel fegato, nella vescica e nel muscolo, come dimostra la media delle concentrazioni rilevate; i risultati ottenuti pertanto sono in accordo con quanto già evidenziato nella specie suina ed in altre specie (avicoli) da altri Autori (Bozzo et al., 2008,

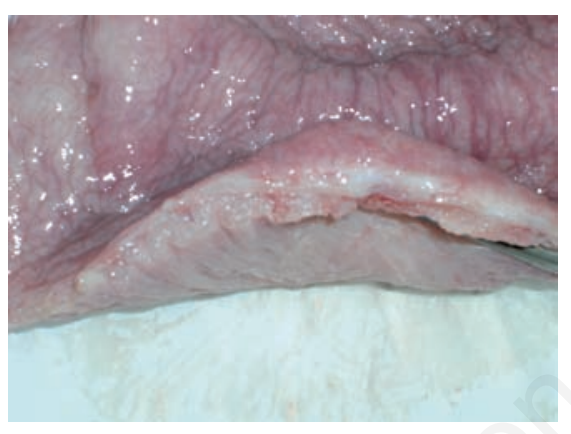

Figura 5. Vescica urinaria: notevole ispessimento della parete.

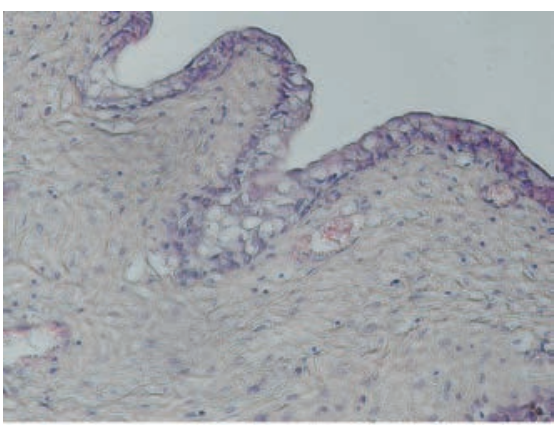

Figura 6. Vescica urinaria: degenerazione dell'epitelio vescicale e vacuolizzazione citoplasmatica (colorazione ematossilina eosina, ingrandimento $40 \mathrm{x}$ ).
2011; Ceci et al., 2007; Milicevic et al., 2007). Tuttavia, poiché i cinghiali oggetto dell'indagine erano stati allevati allo stato brado è necessario ipotizzare che in queste condizioni abbiano comunque ingerito alimenti contaminati da muffe; è noto che i cinghiali, onnivori, pascolatori e grufolatori se allevati allo stato brado, conservano più spiccatamente le loro particolari abitudini alimentari. La possibilità di controllare la produzione primaria, ponendo particolare attenzione alla qualità dei mangimi, e applicando le corrette prassi agricole, così come raccomandato dalle recenti normative, risulta, in tale contesto, una azione preventiva irrealizzabile. È bene evidenziare, inoltre, che le carni di cinghiale vengono spesso utilizzate per la produzione di salumi stagionati; particolare attenzione, quindi, deve essere rivolta alla presenza dell'OTA, come di qualsiasi altro contaminante, nelle carni da trasformare, poiché la stagionatura consente di ottenere, nel prodotto finito, una concentrazione molto più elevata. Sebbene finora non siano stati condotti studi epidemiologici in grado di stabilire quale ruolo possa essere attribuito all'assunzione di carni di cinghiale contaminate da OTA nelle ocratossicosi umane, la presenza della micotossina rilevata rappresenta un pericolo per la salute dell'uomo.

\section{Conclusioni}

I rilievi anatomo-istopatologici effettuati a carico del rene, del fegato e della vescica urinaria mettono in evidenza quanto il cinghiale sia sensibile all'azione dell'OTA e dimostrano quanto sia prioritario approntare sistemi diagnostici da impiegare direttamente nel mattatoio, unitamente ad una rivisitazione sostanziale dei procedimenti ispettivi post-mortem al fine di rendere lo stabilimento di macellazione un osservatorio epidemiologico utile alla corretta gestione della Sanità Pubblica.

\section{Bibliografia}

Abarca M, Accensi F, Bragulat MR, Cabanes FJ, 2001. Current importance of ochratoxin Aproducing Aspergillus spp. J Food Protect 64:903-6.

Bozzo G, Bonerba E, Ceci E, Colao V, Tantillo G, 2011. Determination of ochratoxin A in eggs and target tissues of experimentally drugged hens using HPLC-FLD. Food Chem 126:1278-82.

Bozzo G, Ceci E, Bonerba E, Desantis S, Tantillo G, 2008. Ochratoxin A in laying hens: high-performance liquid chromato- graphy detection and cytological and histological analysis of target tissues. J Appl Poultry Res 17:151-6.

Brown RL, Bhatnagar D, Cleveland TE, Cary JW, 1998. Recent advances in preharvest prevention of mycotoxin contamination. In: Sinha KK, Bhatnagar D, eds. Mycotoxins in agriculture and food safety. Marcel Dekker ed., New York, NY, USA.

Ceci E, Bozzo G, Bonerba E, Di Pinto A, Tantillo G, 2007. Ochratoxin A detection by HPLC in target tissues of swine and cytological and histological analysis. Food Chem 105:364-8.

Commissione Europea, 2006. Regolamento della Commissione del 19 dicembre 2006 che definisce I tenori massimi di alcuni contaminanti nei prodotti alimentari, 1881/2006/CE. In: Gazzetta Ufficiale, L 364, 20/12/2006.

Delage N, D'Harlingue A, Ceccaldi BC, Bompeix G, 2003. Occurrence of mycotoxins in fruit juices and wine. Food Control 14:225-7.

Milicevic D, Verica Juric M, Mandic M, Djordjevic N, 2007. The presence of ochratoxin A residue in blood plasma of slaughtered swine. Zbornik Matice srpske za prirodne nauke 113:55-62. Available from: http://www.doiserbia.nb.rs/img/doi/03524906/2007/0352-49060713055M.pdf

Ministero della Sanità, 1999. Direttive in materia di controllo ufficiale sui prodotti alimentari: valori massimi ammissibili di micotossine nelle derrate alimentari di origine nazionale,comunitaria e Paesi Terzi. In: Gazzetta ufficiale, no. 135, 11/06/1999.

Monaci L, Tantillo G, Palmisano F, 2004. Determination of ochratoxin A in pig tissue by liquid-liquid extraction/partition and high performance liquid chromatography. Anal Bioanal Chem 378:1777-82.

0'Brien E, Dietrich DR, 2005. Ochratoxin A: the continuing enigma. Crit Rev Toxicol 35:33-60.

Peterson SW, 2000. Phylogenetic relationships in Aspergilluus based on rDNA sequence analysis. In: Samson RA, Pitt JI, eds. Integration of modern taxonomic methods for Penicillium and Aspergillus classification. Harwood Academic Pubbl., Amsterdam, Paesi Bassi.

Pfohl-Leszkowicz A, Manderville RA, 2007. Review on Ochratoxin A: an overview on toxicity an carcinogenicity in animals and humans. Mol Nutr Food Res 51:61-99.

Schaaf GJ, Nijmeijer SM, Maas RF, Roestenberg P, De Groene EM, FinkGremmels J, 2002. The role of oxidative stress in the ochratoxin A-mediated toxicity in proximal tubular cells. Biochim Biophys Acta 1588:149-58. 